\title{
Sexuelle Dysfunktionen bei Frauen mit Posttraumatischer Belastungsstörung - ein Übersichtsartikel
}

\author{
Pia Bornefeld-Ettmann Regina Steil \\ Abteilung klinische Psychologie und Psychotherapie, Institut für Psychologie, \\ Goethe Universität Frankfurt Main, Frankfurt/M., Deutschland
}

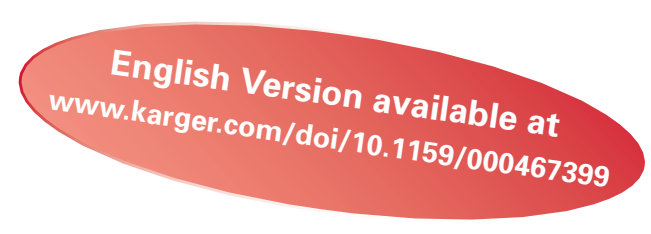

\section{Schlüsselwörter \\ Sexuelle Dysfunktionen · Posttraumatische Belastungsstörung · Sexuelle Gewalt . \\ Sexueller Kindesmissbrauch}

\section{Zusammenfassung}

Sexuelle Dysfunktionen, sowohl klinischer als auch subklinischer Art, sowie andere sexuelle Beschwerden, die mit aversiven Emotionen, Kognitionen und sexuellem Risikoverhalten einhergehen, treten bei Frauen nach sexuellem Missbrauch sehr häufig auf und sind ein stark unterschätztes Problem. Aber auch bei Opfern anderer Arten von Traumatisierung ergeben sich hohe Prävalenzen sexueller Beschwerden. Dies lässt sich lerntheoretisch sowie anhand der Phänomenologie der Posttraumatischen Belastungsstörung (PTBS) an sich erklären. Sexualität ist eine wichtige Quelle von Lebensfreude, und das Fortbestehen sexueller Dysfunktionen kann mit dem Risiko von Einbußen in Lebensqualität und Selbstwertgefühl sowie von partnerschaftlichen Problemen einhergehen. Neben der klinischen Praxis besteht auch in der klinischen Forschung nach wie vor nur ein unzureichendes Wissen bezüglich des Zusammenhangs zwischen sexuellen Dysfunktionen und traumatischen Erfahrungen, vor allem sexueller Art. Im folgenden Artikel werden die Phänomenologie und Diagnostik weiblicher sexueller Dysfunktionen vorgestellt und Folgen von sexueller Gewalterfahrung auf die Sexualität, auch im Kontext einer PTBS, näher erläutert. Zudem werden Vorschläge zur Integration entsprechender Behandlungsbausteine in die Therapie gemacht

(c) 2017 The Author(s). Published by S. Karger GmbH, Freiburg

\begin{abstract}
Keywords
Sexual Dysfunctions · Posttraumatic stress disorder . Sexual violence .

Sexual child abuse

\section{Summary \\ Sexual Dysfunctions in Women with Posttraumatic \\ Stress Disorder - a Review Article}

Sexual dysfunctions, clinical as well as subclinical, and other sexual complaints that accompany aversive emotions, cognitions, and sexual risk behavior are very common among women after experiences of sexual violence and are underestimated as a problem. Anyway, among victims of other kinds of traumatic experiences, a high prevalence of sexual distress is found as well. Explanatory approaches concentrate on learning theory as well as the phenomenology of Posttraumatic Stress Disorder (PTSD) itself. Sexuality is an important source of vitality, and the maintenance of sexual dysfunctions can be accompanied by a risk of impairments in quality of life and self-esteem as well as relationship problems. Aside clinical practice, there is also a lack of knowledge in clinical science regarding the association between sexual dysfunctions and traumatic experiences, especially sexual ones. In this article, the phenomenology and diagnostic instruments of female sexual dysfunctions are presented, and consequences of sexual violence on sexuality, also in the context of PTSD, are further explained. Suggestions are being made on how to include suitable interventions into therapy.
\end{abstract}

\section{KARGER \\ Fax +497614520714

(๖) 2017 The Author(s)
Published by S. Karger GmbH, Freiburg
Open arger

This article is licensed under the Creative Commons AttributionNonCommercial-NoDerivatives 4.0 International License (CC BY-NC ND 4.0) (http://www.karger.com/Services/OpenAccessLicense). Usage no
Pia Bornefeld-Ettmann

Abteilung klinische Psychologie und Psychotherapie, Institut für Psychologie Goethe-Universität Frankfurt am Main

Varrentrappstraße 40-42, 60486 Frankfurt/M., Deutschland

bornefeld-ettmann@psych.uni-frankfurt.de 


\section{Einleitung}

Eine Vielzahl an Untersuchungen zeigt, dass Frauen, die sexuellen Kindesmissbrauch (d.h. sexuelle Gewalterfahrung vor dem 18. Lebensjahr) erlebt haben, sexuelle Dysfunktionen entwickeln [z.B. Stephenson et al., 2014]. Aber auch Frauen, die im Erwachsenenalter sexuelle Gewalt erlebt haben (z.B. Vergewaltigung), leiden danach nicht selten unter sexuellen Dysfunktionen [van Berlo und Ensink, 2000]. Unter sexuellen Dysfunktionen werden im Wesentlichen Beschwerden bzw. Einschränkungen verstanden, die den Erregungszyklus [Kaplan, 1974] bezüglich der Bereiche Lust (Appetenz), Erregung und Orgasmus betreffen. Zusätzlich werden bei Frauen auch Schmerzen im Genital- und Beckenbereich (Dyspareunie) und unwillkürliche Verkrampfungen oder Verspannungen des Beckenbodens und der Vaginalmuskulatur (Vaginismus) hierzu gezählt. Sexuelle Dysfunktionen treten in der Allgemeinbevölkerung bei Frauen und Männern relativ häufig auf [Hoyer, 2013], wobei die Prävalenzen je nach Art der Erhebung sehr stark schwanken, z.B. in Abhängigkeit des Erhebungsinstruments [Hayes et al., 2008b] und der zugrunde gelegten diagnostischen Kriterien (z.B. ob ein klinisch relevanter Leidensdruck als notwendiges Kriterium erfasst wird oder nicht [Reinecke et al., 2006]). Gemäß Lewis et al. [2010] liegen die aus einer Aggregation von 18 internationalen epidemiologischen Studien gezogenen Prävalenzen sexueller Dysfunktionen (vor allem Erregungs- und Appetenzstörungen) in der Allgemeinbevölkerung bei Frauen im Mittel bei etwa $40-45 \%$ und bei Männern bei etwa 20-30\%, wobei die Prävalenzen mit dem Alter stark ansteigen.

Die Prävalenzen von Erregungs-, Appetenz- und Orgasmusschwierigkeiten bei Frauen mit sexuellen Gewalterfahrungen im Erwachsenenalter reichen gemäß einiger Studien bis zu 59\% [z.B. van Berlo und Ensink, 2000]; bei Frauen nach sexuellem Kindesmissbrauch werden sie oft noch höher eingeschätzt [z.B. Leonard und Follette, 2002]. Nach Bartoi und Kinder [1998] unterscheidet sich die Prävalenz sexueller Dysfunktionen bei Frauen jedoch nicht in Abhängigkeit des Alters, in dem die sexuelle Gewalt stattfand. Die insgesamt festgestellten hohen Prävalenzen sexueller Dysfunktionen erlauben nicht den Schluss, dass diese ursächlich auf die Erfahrung sexueller Gewalt zurückzuführen sind. Zum einen handelt es sich ausschließlich um retrospektiv gesammelte Angaben zu sexueller Gewalt, wobei auch sogenannte «false memories» in Bezug auf sexuelle Gewalterfahrungen eine Rolle spielen könnten [Stoffels und Ernst, 2002]. Zum anderen gibt es eine Vielzahl an weiteren Risikofaktoren, die neben sexueller Gewalterfahrung zu der Entwicklung sexueller Dysfunktionen beitragen könnten. Dazu zählen psychische Erkrankungen, vor allem Depressionen, mit Prävalenzen sexueller Dysfunktionen von etwa 50\% [Kennedy und Rizvi, 2009], soziokulturelle Faktoren, wie mangelnde Zuwendung nach der sexuellen Gewalterfahrung oder «Opferbeschuldigung» [Ullman, 2010], und partnerschaftliche Faktoren [Hayes et al., 2008a].

Während sich die Forschung bezüglich eines erhöhten Auftretens sexueller Dysfunktionen bei Frauen mit sexueller Gewalterfahrung oder sexuellem Kindesmissbrauch weitgehend einig ist, wurden bislang nur selten Zusammenhänge zwischen sexuellen Dysfunktionen und der Posttraumatischen Belastungsstörung (PTBS) untersucht. Die PTBS ist gemäß ICD-10 (ICD = International Classification of Diseases) definiert als Reaktion auf ein Ereignis von außergewöhnlicher Bedrohung oder katastrophalem Ausmaß, das bei nahezu jedem tiefgreifende Verzweiflung auslösen würde. Darauf folgen intrusive Erinnerungen oder ein Wiedererleben der Belastung durch verschiedene interne und externe Trigger (Alpträume, Nachhallerinnerungen) sowie Vermeidungsverhalten in Bezug auf Umstände, die dem Ereignis ähneln oder im Zusammenhang damit stehen. Zudem bestehen Erinnerungsschwierigkeiten in Bezug auf einzelne Aspekte des Ereignisses oder anhaltende Symptome einer erhöhten Sensitivität oder Erregung [World Health Organization (WHO), 1992]. Die Kriterien gemäß DSM-5 (DSM = Diagnostic and Statistical Manual of Mental Disorders) werden im Verlauf im Kontext sexueller Dysfunktionen näher genannt. Sehr häufig wurde in den oben genannten Studien zu sexuellen Dysfunktionen bei Frauen nach sexueller Gewalt oder sexuellem Kindesmissbrauch nicht differenziert, ob die Betroffenen an einer PTBS litten oder nicht [O'Driscoll und Flanagan, 2016]. Einzelne Studien belegten jedoch einen Zusammenhang zwischen der Diagnose einer PTBS und sexuellen Dysfunktionen [Letourneau et al., 1996; Haase et al., 2009]. Dabei wurde gezeigt, dass sexuelle Dysfunktionen nicht nur bei PTBS nach sexueller Traumatisierung vorkommen, sondern gehäuft auch bei Opfern anderer Arten von Traumatisierung [z.B. Letourneau et al., 1996].

\section{Phänomenologie und Diagnostik sexueller Dysfunktionen}

Das Ausmaß der Beeinträchtigung durch sexuelle Symptome kann beträchtlich variieren und von leichten, situativ bedingten Symptomen bis zu Beeinträchtigungen mit schwerem Leidensdruck reichen [Beier et al., 2000]. Im folgenden Text sollen die Bezeichnungen vereinheitlicht werden. Mit «sexuellen Dysfunktionen» oder «sexuellen Funktionsstörungen» sind die nach DSM-5 [American Psychiatric Association (APA), 2013] oder ICD-10 [WHO, 1992] diagnostizierten Störungsbilder gemeint. «Sexuelle Beschwerden» bezeichnen die subklinische Ausprägung bzw. zusätzliche Beschwerden, die nicht durch die bisherigen Diagnosen abgedeckt werden, wie sexuelles Risikoverhalten.

Sexuelle Dysfunktionen werden definiert als «Beeinträchtigungen im sexuellen Verhalten, Erleben und in den physiologischen Reaktionen, die eine für beide Partner befriedigende sexuelle Interaktion behindern oder unmöglich machen. Die Schwierigkeiten sind dabei nicht auf organische Ursachen oder eine Fixierung auf unübliche Sexualziele oder -objekte (Paraphilien) zurückzuführen» [aus Kockott und Fahrner, 2000, p. 2]. Die Beschwerden können dabei die eigenständige Diagnose einer sexuellen Funktionsstörung rechtfertigen oder auch vollständig durch ein anderes Störungsbild (vor allem Depression [Michael und O'Keane, 2000]) bzw. die Einnahme von Psychopharmaka und deren Nebenwirkungen [Baldwin et al., 2015] erklärbar sein. Zusätzlich können sexuelle Dys- 
Tab. 1. Diagnosen sexueller Dysfunktionen bei Frauen nach ICD-10, DSM-IV und DSM-5

\begin{tabular}{|c|c|c|c|}
\hline & ICD 10 & DSM-IV & DSM-5 \\
\hline $\begin{array}{l}\text { Sexuelles } \\
\text { Verlangen } \\
\text { und Erregung }\end{array}$ & $\begin{array}{l}\text { F52.0 Mangel oder Verlust von } \\
\text { sexuellem Verlangen; } \\
\text { F52.2 Versagen genitaler } \\
\text { Reaktionen }\end{array}$ & $\begin{array}{l}302.71 \text { Störung mit verminderter } \\
\text { Sexueller Appetenz; } \\
302.72 \text { Störung der sexuellen } \\
\text { Erregung bei der Frau }\end{array}$ & $\begin{array}{l}302.72 \text { weibliche Störung } \\
\text { des sexuellen Interesses / der } \\
\text { Erregung }\end{array}$ \\
\hline $\begin{array}{l}\text { Sexuelle } \\
\text { Aversion }\end{array}$ & $\begin{array}{l}\text { F52.1 sexuelle Aversion und } \\
\text { mangelnde sexuelle Befriedigung; } \\
.10 \text { sexuelle Aversion; } \\
.11 \text { mangelnde sexuelle } \\
\text { Befriedigung }\end{array}$ & $\begin{array}{l}302.79 \text { Störung mit sexueller } \\
\text { Aversion }\end{array}$ & \\
\hline Orgasmus & F52.3 Orgasmusstörung & $\begin{array}{l}302.73 \text { weibliche Orgasmus- } \\
\text { störung }\end{array}$ & $\begin{array}{l}302.73 \text { weibliche Orgasmus- } \\
\text { störung }\end{array}$ \\
\hline Schmerz & $\begin{array}{l}\text { F52.5 nichtorganischer } \\
\text { Vaginismus; } \\
\text { F52.6 nichtorganische } \\
\text { Dyspareunie }\end{array}$ & $\begin{array}{l}\text { 306.51 Vaginismus } \\
\text { 302.76 Dyspareunie }\end{array}$ & $\begin{array}{l}302.76 \text { genital-pelvine } \\
\text { Schmerz- und } \\
\text { Penetrationsstörung }\end{array}$ \\
\hline \multirow[t]{2}{*}{$\begin{array}{l}\text { Sonstige } \\
\text { Diagnosen }\end{array}$} & $\begin{array}{l}\text { F52.7 gesteigertes sexuelles } \\
\text { Verlangen; }\end{array}$ & & \\
\hline & $\begin{array}{l}\text { F52.8 sonstige sexuelle Funktions- } \\
\text { störungen, nicht verursacht durch } \\
\text { eine organische Störung oder } \\
\text { Krankheit; } \\
\text { F52.9 nicht näher bezeichnete } \\
\text { sexuelle Funktionsstörungen, nicht } \\
\text { verursacht durch eine organische } \\
\text { Störung oder Krankheit }\end{array}$ & $\begin{array}{l}\text { substanz-/ } \\
\text { medikamenteninduzierte } \\
\text { sexuelle Dysfunktion }\end{array}$ & $\begin{array}{l}\text { substanz-/ } \\
\text { medikamenteninduzierte } \\
\text { sexuelle Dysfunktion; } \\
302.79 \text { sonstige sexuelle } \\
\text { Dysfunktion; } \\
302.70 \text { nicht näher bezeichnete } \\
\text { sexuelle Dysfunktion }\end{array}$ \\
\hline
\end{tabular}

funktionen auch somatische Ursachen haben oder durch diese aufrechterhalten werden, z.B. als Folge einer Operation [Raina et al., 2007], was bezüglich einer Diagnosestellung berücksichtigt werden muss.

In der ICD-10 werden unter F52 «sexuelle Funktionsstörungen, nicht verursacht durch eine organische Störung oder Krankheit» aufgeführt; dabei gibt es geschlechtsspezifische und geschlechtsübergreifende Störungsbilder. Die Beschreibungen und Unterteilungen der Störungsbilder unterscheiden sich zwischen ICD-10, DSM-IV und DSM-5 in einigen Punkten beträchtlich [z.B. Ehret und Berking, 2013]. Im Wesentlichen wurden im DSM-5 klinische Erfahrungen in Bezug auf den Verlauf weiblicher sexueller Erregung berücksichtigt, indem Erregung nicht mehr ausschließlich in Bezug auf genitale und nichtgenitale Reaktionen, sondern auch auf psychologische Reaktionen («Lust bekommen») aufgenommen wurde.

Im DSM-5 wird neben der Ausprägung der sexuellen Symptomatik (A-Kriterium) auch ein zeitlicher Bezugsrahmen von 6 Monaten (B-Kriterium), ein von Patientenseite geäußerter Leidensdruck (C-Kriterium) sowie der Ausschluss alternativer Erklärungsmöglichkeiten für die Symptomatik (D-Kriterium) gefordert. Dies macht die Diagnosestellung oftmals schwierig, da die menschliche Sexualität durch verschiedene Einflüsse beeinträchtigt werden kann. Dazu zählen neben einer weitgehend normalen Abnahme von sexueller Aktivität und Lust im Verlauf einer längeren Beziehung bzw. mit voranschreitendem Alter auch Lebensabschnitte mit erhöhtem Stress (z.B. Elternschaft kleinerer Kinder, kritische Lebensereignisse). Hinzukommend wurde auch der Einfluss ver- schiedener Medikamente, wie Serotonin-Wiederaufnahmehemmer und Serotonin-Noradrenalin-Wiederaufnahmehemmer, auf das sexuelle Funktionsniveau nachgewiesen [Baldwin et al., 2015]. Nicht zuletzt stellen auch verschiedene andere psychische Störungen einen Einflussfaktor hinsichtlich des sexuellen Befindens dar, wobei vor allem depressive Erkrankungen zu nennen sind [Michael und O'Keane, 2000]. Tabelle 1 zeigt die Diagnosen sexueller Dysfunktionen nach ICD 10, DSM-IV und DSM-5.

\section{Fallbeispiel 1:}

Eine 24-jährige PTBS-Patientin erzählt von stechenden Schmerzen in der Vagina sowie im Beckenbodenbereich, die sie seit einer Vergewaltigung im 14. Lebensjahr habe. Sie würden auftreten, sobald irgendetwas in die Scheide eingeführt werden soll (z.B. beim Frauenarzt oder bei der Nutzung von Tampons); manchmal spürt sie diese Schmerzen bereits beim Gedanken daran. Der Gynäkologe fand keine organische Ursache für die Schmerzen. Aktuell sei sie nicht in einer Beziehung, die letzte sei vor 2 Jahren in die Brüche gegangen. Im letzten Jahr habe sie keinen sexuellen Verkehr gehabt, da sie große Angst vor Schmerzen im Genitalbereich habe. Zuletzt habe sie vor etwa 13 Monaten einen versuchten Geschlechtsverkehr unterbrochen, da die Schmerzen unerträglich waren. Sie fühle sich einsam, wolle aber auch keine Beziehung eingehen, weil dann wahrscheinlich Sex von ihr verlangt werden würde. Darunter leide sie sehr stark, da sie glaube, niemals eine eigene Familie haben und deshalb niemals ein erfülltes Leben führen zu können, was sie sehr traurig mache. 
Tab. 2. Prävalenzen sexueller Dysfunktionen bei Frauen

\begin{tabular}{|c|c|c|}
\hline Sexuelle Dysfunktion & $\begin{array}{l}\text { Prävalenzen im Rahmen } \\
\text { der gynäkologischen } \\
\text { Sprechstunde } \\
(\mathrm{N}=4097)^{*}\end{array}$ & $\begin{array}{l}\text { Prävalenzen in der } \\
\text { amerikanischen } \\
\text { Allgemeinbevölkerung } \\
(\mathrm{N} \approx 28000)^{* *}\end{array}$ \\
\hline Störungen der sexuellen Appetenz & $9,3-15,03 \%$ & $7,4-12,3 \%$ \\
\hline Erregungsstörungen & $0,6 \%$ & $3,3-7,5 \%$ \\
\hline Orgasmusstörungen & $0,6 \%$ & $3,4-5,8 \%$ \\
\hline Vaginismus & $1 \%$ & - \\
\hline Dyspareunie & $20 \%$ & - \\
\hline
\end{tabular}

In Publikationen zu Prävalenzen von sexuellen Dysfunktionen oder in empirischen Studien werden Bezeichnungen der Beschwerden oft synonym oder wenig differenziert verwendet, und lange Zeit lagen keine oder unzureichend validierte Erhebungsinstrumente vor, was auch die erheblichen Schwankungen in Bezug auf die Prävalenzen erklärt [Hayes et al., 2008b]. Eine Übersicht über die Prävalenzen sexueller Dysfunktionen bei Frauen, bei denen neben der Symptomatik an sich auch der Leidensdruck erhoben wurde, ist in Tabelle 2 dargestellt, wobei die Angaben nach Altersgruppe variieren.

Trotz der hohen Prävalenzen werden sexuelle Beschwerden bzw. Dysfunktionen innerhalb der Psychotherapie nach wie vor nur wenig berücksichtigt [Hoyer, 2013]. Dies hat unterschiedliche Gründe, wie Vorbehalte seitens der Therapeuten, ein vermeintlich zu schambesetztes Thema anzusprechen, oder unklare Zuständigkeitsbereiche. Zudem werden sexuelle Dysfunktionen häufig als sekundäres Problem angesehen und deshalb in der Behandlung nicht berücksichtigt [Hoyer, 2013].

Im Folgenden sollen einige Erhebungsinstrumente vorgestellt werden. In klinischen Studien wird sehr häufig der «Female Sexual Distress Index» (FSFI-d) [Berner et al., 2004] eingesetzt. Dabei handelt es sich um ein Selbstbeurteilungsinventar zur Erfassung sexueller Beschwerden in den Bereichen «Lust», «Erregung», «Lubrikation», «Orgasmus», «Befriedigung» und «Schmerzen». Das Inventar kann als Screening-Instrument verstanden werden. Der FSFI erfasst allerdings weder Leidensdruck noch alternative Erklärungen der Beschwerden (z.B. durch Einnahme eines Medikaments), zudem bezieht er sich nur auf einen Zeitraum von 4 Wochen und kann deshalb nicht als verlässliches Diagnoseinstrument genutzt werden.

Der «Kurzfragebogen zu sexuellen Funktionsstörungen» [Hoyer und Jahnke, 2014] erfasst die Störungsbereiche sexueller Dysfunktionen für Frauen und Männer, indem grob die Diagnosekriterien abgefragt werden, wobei auch der Leidensdruck berücksichtigt wird. Alternative Erklärungen für sexuelle Beschwerden, wie die Einnahme von Medikamenten, Krankheiten oder andere Ursachen gemäß DSM-5-Kriterium D, werden nicht erfasst. Es handelt sich um ein kurzes und deshalb für die klinische Praxis gut geeignetes Screening-Instrument zur Erfassung des Vorliegens sexueller Beschwerden bzw. Dysfunktionen, die zur validen Diagnosestellung im weiteren Verlauf genauer exploriert werden müssen.

Das einzige im deutschsprachigen Raum bekannte diagnostische Interview zur Erfassung sexueller Dysfunktionen ist das
«Strukturierte Interview zu sexuellen Funktionsstörungen» (SISEX) [Hoyer et al., 2014]. Dazu liegt eine Forschungsversion vor, die im Rahmen einer großen randomisierten und kontrollierten Psychotherapiestudie zur Behandlung der PTBS speziell für PTBS-Patientinnen entwickelt wurde [Bornefeld-Ettmann und Hoyer, 2014]. Das SISEX erfasst die Bereiche «Störung des sexuellen Interesses und der Erregung», «Orgasmusstörung» sowie die «genital-pelvine Schmerz- und Penetrationsstörung» gemäß DSM5. Mithilfe des SISEX können sexuelle Beschwerden per Fremdbeurteilung erhoben und diagnostiziert werden, wobei nicht nur das Vorliegen der Symptome und deren Ausprägung abgefragt, sondern auch Kriterien B und C (zeitlicher Bezugsrahmen sowie Leidensdruck) erfasst werden. Zudem werden alternative Erklärungsmöglichkeiten der Symptomatik sowie mögliche auslösende Faktoren erfasst. Abschließend können ein Subtyp (generalisiert vs. erworben) sowie ein Schweregrad bestimmt werden.

\section{Folgen von sexueller Gewalt auf die Sexualität}

Die schädlichen Folgen sexuellen Kindesmissbrauchs auf das psychische Wohlbefinden im Erwachsenenalter wurden in vielen empirischen Studien nachgewiesen [Maniglio, 2009], aber auch die Folgen auf die Sexualität wurden bereits mehrfach untersucht [z.B. Stephenson et al., 2014]. Studien zu den Folgen sexueller Gewalterfahrungen im Erwachsenenalter und den Auswirkungen auf die Sexualität sind selten. Bei Bartoi und Kinder [1998] unterschieden sich die Häufigkeiten sexueller Dysfunktionen nicht in Abhängigkeit des Alters, in dem die sexuelle Gewalterfahrung stattfand. Auch Chapman [1989] zeigte eine Häufung sexueller Dysfunktionen bei Frauen, die im Erwachsenenalter vergewaltigt wurden. Daraus ergibt sich der Schluss, dass das Auftreten sexueller Dysfunktionen unabhängig davon ist, in welchem Lebensalter die sexuelle Gewalt stattfand. Dazu stehen jedoch genauere Untersuchungen aus, z.B. darüber, wie sich die Folgen chronischer versus einmaliger sexueller Gewalterfahrungen unterscheiden oder welche sexuellen Funktionen im Speziellen betroffen sind.

Das Symptombild der Frauen, die sexuelle Gewalt erleben mussten, kann sehr komplex sein, da neben sexuellen Dysfunktionen auch sexuelle Beschwerden auftreten können, die sich auf kognitive, emotionale und behaviorale Prozesse beziehen, wie Vermei- 
dung von Sexualität aufgrund befürchteter intrusiver Symptome oder Risikoverhalten in Bezug auf Sexualität zur Selbstbestrafung oder zum Stressabbau. Eine Vielzahl an Studien hat eine Häufung riskanter sexueller Verhaltensweisen, wie häufig wechselnde Sexualpartner, einen frühen Beginn sexueller Aktivität und ungeschützten Geschlechtsverkehr, bei Frauen nach sexuellem Kindesmissbrauch belegt [Senn et al., 2008]. Allerdings deuten andere Befunde [z.B. Büttner et al., 2014] auf ein ausgeprägtes Vermeidungsverhalten in Bezug auf Sexualität bei PTBS-Patientinnen hin, sodass sich kein einheitliches Bild ergibt. Es ist denkbar, dass sexuelles Risikoverhalten mit der oft bei komplex traumatisierten Frauen vorliegenden Borderline-Persönlichkeitsakzentuierung bzw. BorderlinePersönlichkeitsstörung zusammenhängt. Eine Komorbidität der beiden Störungsbilder ist häufig und geht mit erhöhter Symptombelastung einher [Scheiderer et al., 2015].

Es ist wichtig, auch subklinische bzw. durch ein anderes Störungsbild bedingte sexuelle Beschwerden, die nicht die Diagnosekriterien einer sexuellen Dysfunktion erfüllen, in der Behandlung zu berücksichtigen, da Beschwerden sehr heterogen auftreten können [Hofmann, 2015]. Auch diese können zu Beeinträchtigungen im Wohlbefinden und einem Leiden führen [Stephenson und Meston, 2015] und für die Aufrechterhaltung von Traumafolgestörungen relevant sein. Als Beispiel können hier mit Sexualität verbundene negative Selbstbewertungen [z.B. Meston et al., 2006] genannt werden. Studien zeigten, dass sich Frauen mit sexueller Gewalterfahrung als weniger romantisch und leidenschaftlich wahrnehmen [Meston et al., 2006] und dass sie ein geringeres Selbstwertgefühl in Bezug auf ihre Sexualität haben [Shapiro und Schwarz, 1997] als Frauen ohne solche Erfahrungen, was gemäß van Bruggen et al. [2006] teilweise einen Einfluss auf erneute sexuelle Gewalterfahrungen im späteren Leben (Reviktimisierung) haben kann. Denkbar ist, dass betroffene Frauen sich aufgrund der negativen Selbstbewertung in Bezug auf die eigene Sexualität häufig nicht zutrauen, in sexuellen Situationen Grenzen zu setzen, oder grobes Verhalten eines Partners eher akzeptieren, da sie annehmen, es nicht anders zu verdienen. Diese Faktoren sind spekulativ, jedoch erscheinen sie plausibel, um die hohen Raten an Reviktimisierung [Messmann und Long, 1996] zu erklären.

Als sekundäre Probleme zu sexuellen Dysfunktionen können sich andauernde partnerschaftliche Probleme, ein unerfüllter Kinderwunsch, ein geringes Kontrollempfinden in Bezug auf den eigenen Körper und insgesamt eine Einschränkung der Lebensqualität ergeben [Beier et al., 2000].

\section{Fallbeispiel 2:}

Eine Patientin mit PTBS nach sexuellem Missbrauch stellt sich mit dem Wunsch einer ambulanten Traumatherapie vor. Auf die Frage, warum sie ihre Traumasymptomatik zum jetzigen Zeitpunkt angehen wolle, berichtet sie, seit $1 \mathrm{Jahr}$ in einer an sich glücklichen Beziehung zu leben. Jedoch habe sie seit dem sexuellen Missbrauch im Alter von 12 Jahren extreme Angst vor Sexualität und deshalb bislang nicht mit dem Freund schlafen können. Er habe ihr jetzt ein Ultimatum gestellt, wonach er sich von ihr trennen werde, wenn sie die Problematik nicht in den Griff bekommt.
Obwohl die Häufung sexueller Symptome bei Frauen, die sexuelle Gewalt erlebt haben, inzwischen bekannt ist, mangelt es an theoretischen Erklärungsmodellen zum Zusammenhang zwischen sexueller Gewalterfahrung und sexuellen Dysfunktionen [Leonard und Follette, 2002]. Zwei Erklärungsansätze sollen im Folgenden vorgestellt werden.

In ihrem kontextuellen behavioralen Modell beschreiben Polusny und Follette [1995] die Rolle von auf Erfahrung beruhender Vermeidung, sogenannter «experiential avoidance». «Experiential avoidance» wird als Prozess des Widerwillens beschrieben, schmerzhafte Gedanken, Gefühle und Erinnerungen zu erleben, die mit sexueller Gewalt oder anderen traumatischen Erfahrungen assoziiert sind [Hayes et al., 1996]. Die Vermeidung unangenehmer innerlicher Vorgänge folgt dabei lerntheoretisch der 2-Faktoren-Theorie [Mowrer, 1960], die annimmt, dass zunächst Angst klassisch konditioniert und Vermeidungsverhalten durch operante Konditionierung aufrechterhalten wird. Die Erfahrung sexueller Gewalt führt dementsprechend dazu, dass zukünftige sexuelle Situationen als konditionierter Reiz zu Angstreaktionen führen und vermieden werden. Angstreaktionen führen zusätzlich zu psychophysiologischen Veränderungen, die sich negativ auf das sexuelle Funktionsniveau auswirken [Yehuda et al., 2015].

\section{Fallbeispiel 3:}

Eine Patientin mit PTBS nach sexuellem Missbrauch berichtet von häufig wechselnden Sexualpartnern. Dies erscheint paradox, da sie von sexueller Unlust und Erregungsschwierigkeiten berichtet. Auf die Frage, warum sie so schnell sexuellen Kontakten zustimme, gibt sie an, dies zu tun, damit kein Konflikt mit einem Mann entsteht, der an ihr sexuell interessiert ist. Wenn sie möglichst schnell in den Sex einwillige, reduziere sich ihre Angst vor einer potenziellen Bedrohung, die bestehen könnte, wenn sie dem Mann einen Korb gibt.

Eine zweite Theorie ist die Emotionstheorie, die postuliert, dass frühe Missbrauchserfahrungen die emotionale Entwicklung beeinträchtigen, woraus maladaptive Bewältigungsstrategien resultieren können [Leonard und Follette, 2002; zitiert nach Greenberg et al., 2002]. Die Emotionstheorie besagt, dass emotionaler Schmerz durch ein Trauma wie sexuelle Gewalt ausgelöst werden und adaptiv wirken kann, indem er den Betroffenen vermittelt, dass ein schlimmes Ereignis geschehen ist, das in der Zukunft vermieden werden sollte. Eine chronische Vermeidung emotionaler Schmerzen ist maladaptiv und kann zum Verlust primärer Emotionen führen, was die Unfähigkeit mancher Opfer sexuellen Missbrauchs, sexuelle Lust für ihren Partner oder Intimität zu empfinden, erklären kann [Leonard und Follette, 2002]. Extrem negative traumatische Erfahrungen können in der Entwicklung maladaptiver emotionaler Schemata, die komplexe kognitive, affektive, motivationale und Handlungskomponenten enthalten, resultieren [Greenberg et al., 2002]. Diese Schemata können in späteren Beziehungen aktiviert werden, was dann zur Auslösung von negativen Erwartungen und von Mustern physiologischer Erregung, die mit der sexuellen Gewalterfahrung verknüpft sind, führt [Leonard und Follette, 2002]. Entsprechend erscheint der Erklärungsansatz plausibel, dass 
Opfer von sexueller Gewalt sexuelle Beschwerden und Dysfunktionen erleben, da bei intimem körperlichem Kontakt maladaptive Schemata und damit verbundene Gefühle der Bedrohung und der Angst aktiviert werden. So können auch riskante und paradox erscheinende sexuelle Verhaltensweisen, z.B. promiskuitives Verhalten, durch diese Theorie erklärt werden. Aufgrund durch aktivierte Schemata hervorgerufener Gefühle von Bedrohung werden verschiedene Verhaltensweisen gezeigt, die die Bedrohung abmildern sollen, z.B. auch das schnelle Einwilligen in einen sexuellen Kontakt. Dafür sprechen Befunde von Lacelle et al. [2012], die zeigten, dass gravierendere Formen von sexuellem Kindesmissbrauch (mit versuchter oder durchgeführter Penetration) zu einer erhöhten Wahrscheinlichkeit für späteres sexuelles Risikoverhalten führen.

\section{Fallbeispiel 4:}

Eine Patientin mit PTBS nach sexuellem Missbrauch berichtet von vielfachen Erfahrungen von Reviktimisierung. Sie sei im Kindesalter durch den Onkel sexuell missbraucht worden, später sei dies mehrfach durch verschiedene Partner geschehen, kürzlich habe sie jedoch auch ein Bekannter vergewaltigt. Sie berichtet, sich im Beisein von Männern eigentlich sehr unwohl zu fühlen und deshalb in der Vergangenheit sehr viel Alkohol konsumiert zu haben, um ihre Angst zu dämmen. Sie sei dann auch besser gelaunt und bekomme dann mehr positives Feedback von anderen. Es gefalle ihr dann, wenn Männer ihr sagen, dass sie hübsch sei, und ihr Aufmerksamkeit schenken. Sie habe den Eindruck, dass etwas an ihr nicht stimmen könne, da sie von Männern so häufig sexuell ausgenutzt werde.

\section{Sexuelle Dysfunktionen und PTBS}

In Anlehnung an die beiden geschilderten Erklärungsmodelle zum Zusammenhang zwischen sexueller Gewalterfahrung und sexuellen Dysfunktionen erscheint es nachvollziehbar, dass zunächst die Art der sexuellen Gewalt für die Entwicklung sexueller Dysfunktionen mitverantwortlich ist, unabhängig von der Ausbildung einer PTBS. Jedoch gibt es zahlreiche Studien, die eine Häufung sexueller Dysfunktionen bei PTBS-Patienten belegen. Dies trifft auf weibliche und männliche Patienten sowie bei verschiedenen Arten von Traumaerfahrungen zu [z.B. Yehuda et al., 2015]. Es ist denkbar, dass die Befunde konfundiert sind, da Opfer von sexuellem Kindesmissbrauch und sexueller Gewalt im Erwachsenenalter sehr häufig eine PTBS entwickeln [Elklit und Christiansen, 2010], was jedoch in zahlreichen Studien nicht erfasst wurde. Möglicherweise ist das Vorliegen einer PTBS tatsächlich im Sinne einer moderierenden Wirkung ausschlaggebend ist für die Entwicklung sexueller Dysfunktionen [Letourneau et al., 1996], eventuell durch PTBS-spezifische Symptome wie kognitive Verzerrungen und Hyperarousal.

Dem wiedersprechen jedoch Befunde von Arbanas [2010], der das gleiche Ausmaß sexueller Dysfunktionen bei nicht sexuell traumatisierten Männern, die eine PTBS entwickelten, im Vergleich zu nicht sexuell traumatisierten Männern, die keine PTBS entwickelten, fand. Demnach war das Vorliegen einer PTBS nicht ausschlaggebend für das Vorliegen sexueller Dysfunktionen. Da in der Stu- die jedoch nur Männer untersucht wurden, ist unklar, ob sich dies auf Frauen übertragen lässt. In einer populationsbasierten Untersuchung in Australien [Najman et al., 2005] litten männliche Opfer von sexuellem Kindesmissbrauch seltener an sexuellen Dysfunktionen als weibliche. Also scheint es, dass sich sexueller Kindesmissbrauch bei Männern weniger auf das sexuelle Funktionsniveau auswirkt als bei Frauen. Zu beachten ist, dass die PTBS auch unabhängig von der Art des Traumas zu Einschränkungen im sexuellen Funktionieren führen kann. Der berichtete Geschlechterunterschied könnte somit auch durch die generell höhere Prävalenz der PTBS bei Frauen im Vergleich zu Männern erklärt werden [Kessler et al., 2005]. Hierzu stehen jedoch weitere Untersuchungen aus.

Der Zusammenhang zwischen sexuellen Funktionsstörungen und der Art der Traumatisierung bei PTBS-Patientinnen wurde nur in wenigen Studien systematisch untersucht. Eine Ausnahme stellt eine Querschnittsstudie dar, in der 483 PTBS-Patienten (25,5\% Männer, 74,5\% Frauen) hinsichtlich somatoformen Störungen untersucht wurden [Tagay et al., 2004]. In Bezug auf die Auswirkung der Art der Traumatisierung ergab die Studie, dass PTBSPatienten mit interpersonellen Traumata (sexuelle oder körperliche Gewalt) signifikant häufiger über sexuelle Beschwerden (Schmerzen beim Geschlechtsverkehr, sexuelle Gleichgültigkeit sowie unangenehme Empfindungen im oder am Genitalbereich) klagten als PTBS-Patienten mit traumatisch erlebten Naturkatastrophen, Krankheiten oder Unfällen. Zudem klagten Frauen häufiger als Männer über sexuelle Beschwerden.

Vor dem Hintergrund fehlender Studien, die einen Zusammenhang zwischen der Art des Indextraumas (das als am traumatischsten empfundene Ereignis) bei PTBS-Patientinnen und dem Auftreten sexueller Funktionsstörungen belegen, untersuchten Haase et al. [2009] eine Stichprobe mit 89 PTBS-Patientinnen. Die Studie ergab, dass 63,3\% der PTBS-Patientinnen mit nichtsexueller Traumatisierung und $85 \%$ der sexuell traumatisierten PTBS-Patientinnen sexuelle Dysfunktionen in mindestens einem der vier erfassten Bereiche der sexuellen Funktionsfähigkeit (Appetenz, Erregungsfähigkeit, Orgasmus- und Lubrikationsfähigkeit) hatten. PTBS-Patientinnen mit sexuellem Indextrauma hatten unter Berücksichtigung komorbider depressiver Erkrankungen im Vergleich zu PTBS-Patientinnen mit nichtsexuellem Indextrauma ein 3,8-fach erhöhtes Risiko, sexuelle Dysfunktionen zu entwickeln [Haase et al., 2009]. Zusammenfassend liefert diese Studie Nachweise sowohl für eine hohe Prävalenz von Einschränkungen der sexuellen Funktionsfähigkeit bei PTBS-Patientinnen mit unterschiedlichen Indextraumata als auch für eine erhöhte Wahrscheinlichkeit sexueller Funktionsbeeinträchtigungen bei sexuellen im Vergleich zu nichtsexuellen Indextraumata bei PTBS.

Da lerntheoretische Modelle aus den oben genannten Gründen keine ausreichende Erklärung für das gehäufte Auftreten sexueller Dysfunktionen auch bei nicht sexuell traumatisierten Patienten bieten, ist es denkbar, dass das Störungsbild der PTBS selbst mit der Entstehung von sexuellen Funktionsstörungen bei PTBS-Patienteninnen in Zusammenhang steht. Dabei erscheinen vor allem die Kriterien C, D und E als plausible Einflussfaktoren auf das sexuelle Funktionieren bei nichtsexueller Traumatisierung relevant. 
O'Driscoll und Flanagan [2016] haben die DSM-5-Kriterien der PTBS in Bezug auf Einflussfaktoren sexueller Dysfunktionen und Beschwerden untersucht. Deren grobe Aufstellung wird im Folgenden erweitert:

Gemäß Kriterium A muss eine Konfrontation mit einem traumatischen Ereignis stattgefunden haben. Die Erfahrung von sexueller Gewalt führt dabei relativ häufig (z.B. bei $45 \%$ nach einer Vergewaltigung) zu der Entstehung einer PTBS [Elklit und Christiansen, 2010]. Ebenfalls häufig ist eine PTSD-Erkrankung nach persönlicher Gefährdung durch Kriegsereignisse (in 50-65\% der Fälle), nach Verkehrsunfällen (3-11\%) und Naturkatastrophen (5\%) [Maercker und Michael, 2009]. Gemäß Sarwer und Durlak [1996] ist die Art der sexuellen Gewalterfahrung ein wichtiger Prädiktor für spätere sexuelle Dysfunktionen bei Frauen, wobei die wichtigsten Parameter der Einsatz von physischer Gewalt sowie ob eine Penetration stattfand sind. Über die direkten Auswirkungen von anderen Arten von Traumatisierung auf das sexuelle Funktionsniveau, unabhängig von der Diagnose einer PTBS, ist bislang wenig bekannt.

Das Kriterium B umfasst intrusive Symptome. Sexuelle Situationen könnten gemäß des Traumagedächtnisses [Foa und Kozak, 1986] zu intrusiven Symptomen führen, die dann aversive Emotionen und körperliche Reaktionen hervorrufen, die wiederum die sexuellen Funktionen beeinträchtigen [Barlow, 1986]. Aus lerntheoretischer Sicht erscheint es logisch, dass bei sexueller Traumatisierung die sexuelle Situation an sich zu Intrusionen führen kann, Sexualität daher noch eher vermieden wird als bei anderen Arten von Traumatisierung und somit das Risiko der Entwicklung einer sexuellen Dysfunktion erhöht ist. Dies deckt sich mit Befunden von Büttner et al. [2014], die zeigten, dass Frauen mit sexueller Gewalterfahrung häufiger an «hyposexuellen Störungen» (operationalisiert als Aversion gegen Berührungen und Vermeidung von Sexualität) litten als nichtsexuell Traumatisierte. Jedoch erscheinen auch negative Auswirkungen intrusiver Symptome nach anderen Arten von Traumatisierung auf die Sexualität plausibel, wie z.B. Ängste nach physischer Gewalterfahrung davor, dass bestimmte Körperteile berührt werden, intrusiv beeinflusste Schmerzen nach einem traumatischen Verkehrsunfall oder nach Kriegserfahrungen [Tran et al., 2015]. Starke emotionale Reaktionen können zudem zu dissoziativen Zuständen führen [Schauer und Elbert, 2010], die dann wiederum die Angst vor Sexualität aufrechterhalten, unter anderem auch, weil keine korrigierenden Erfahrungen gemacht werden können oder keine Diskrimination zur Traumasituation vorgenommen werden kann [van Berlo und Ensink, 2000; Hansen et al., 2012]. Es gibt sogar Befunde, die zeigen, dass sich bei Frauen, die anhaltendem sexuellem Kindesmissbrauch ausgesetzt waren, kortikale Veränderungen in der Repräsentation genital-somatosensorischer Felder ergeben, die zunächst auf die sensorische Verarbeitung des Missbrauchs abschwächend wirken, im Verlauf jedoch aufgrund der geringeren Repräsentation genital-somatosensorischer Felder zu sexuellen Dysfunktionen im Erwachsenenalter führen [Heim et al., 2013].

Das Kriterium C erfasst mit traumabezogenen internen oder externen Triggern verbundenes Vermeidungsverhalten. Es ist denk- bar, dass Sexualität an sich als externer Trigger wirkt und deshalb vermieden wird (siehe oben «experiential avoidance» [Hayes et al., 1996]). Ebenso ist nicht auszuschließen, dass interne Trigger in sexuellen Situationen vermieden werden, da Symptome von Erregungserhöhung (schneller Atem, erhöhte Frequenz des Herzschlags) allgemein als aversiv wahrgenommen werden [Yehuda et al., 2015; Letourneau et al., 1996]. Emotionales Abstumpfen (emotional numbing) tritt nicht nur nach sexueller Traumatisierung, sondern auch nach anderen Arten von Traumaerfahrungen auf und kann negative Auswirkungen auf das sexuelle Funktionsniveau haben, da angenehme Erfahrungen nicht wahrgenommen werden, unter anderem Gefühle von Nähe und Liebe zum Partner [Yehuda et al., 2015]. Die Vermeidung von Emotionen erklärt auch das häufige Auftreten von sexuellem Risikoverhalten, wie Substanzmissbrauch zur Betäubung oder Promiskuität zur Vermeidung von Intimität [z.B. Houck et al., 2010; Senn et al., 2008].

Das Kriterium D beschreibt eine Veränderung von Kognitionen und Stimmung. Es erscheint plausibel, dass sich aus Traumatisierung - sowohl sexueller Natur als auch aus anderen Arten von Trauma (physische Gewalt, Gefangenschaft, Folter) - negative Annahmen in Bezug auf Sexualität entwickeln (sogenannte Stuck Points [Resick und Schnicke, 1993]), die mit aversiven Emotionen einhergehen. Diese negativen Annahmen können z.B. die eigene Person betreffen («Ich bin es nicht wert, dass jemand zu mir zärtlich ist»; «Ich darf keinem trauen, muss immer die Kontrolle behalten») und sich negativ auf das sexuelle Selbstkonzept auswirken. Das Zusammenspiel aus sexueller Gewalterfahrung, sexuellen Dysfunktionen und negativem sexuellem Selbstkonzept wurde in einigen Studien belegt [z.B. Meston et al., 2006]. Daneben können sich auch generelle Annahmen über Sexualität ergeben (z.B. «Sex ist ekelhaft und immer gewalttätig»), die wiederum eine direkte Auswirkung auf das sexuelle Funktionsniveau haben. Dies ist potenziell auch nach anderen Arten von Traumatisierung und daraus folgender Traumafolgestörung der Fall; z.B. kann es zu anhaltender Selbstabwertung («Ich war schuld an dem Unfall, ich verdiene nichts Gutes mehr») oder verzerrter Annahmen über andere Menschen («Wenn ich schwäche zeige, werde ich ausgenutzt») kommen, was indirekt Auswirkungen auf das sexuelle Funktionieren haben kann.

Neben den kognitiven Veränderungen berichten einige Frauen nach sexueller Gewalterfahrung von einem anhaltenden Gefühl von Beschmutztheit, das mit Ekel vor dem eigenen Körper sowie Schamgefühlen einhergeht [Jung et al., 2011]. Es erscheint plausibel, dass dies ebenfalls zur Vermeidung von Sexualität beiträgt und, da der eigene Körper als abstoßend wahrgenommen wird, auch Sexualität als unangenehm empfunden wird, was wiederum das Auftreten sexueller Dysfunktionen stark begünstigt.

Das Kriterium E beschreibt Veränderungen in Arousal und Reaktivität, wie Konzentrationsprobleme, Anspannung und Reizbarkeit. Bei erhöhter Grundanspannung sind biologische Prozesse beeinträchtigt, die für sexuelle Funktionen wichtig sind, z.B. die optimale Aktivierung des sympathischen Nervensystems, was zu Dysfunktionen führen kann [Yehuda et al., 2015]. Zudem geht die psychische Anspannung zumeist auch mit einer somatisch erhöh- 
ten Anspannung einher, die wiederum die Wahrscheinlichkeit von genitalen Schmerzen erhöht [Payne et al., 2005]. Dies kann dann weiterhin verstärkend auf negative Grundannahmen bezüglich Sexualität sowie auf Vermeidungsverhalten wirken.

\section{Behandlung sexueller Dysfunktionen im Rahmen der PTBS-Therapie}

Studien, die sich mit der Behandlung sexueller Dysfunktionen im Rahmen einer Behandlung der PTBS befassen bzw. die sexuelle Symptome in diesem Kontext erfassen, sind sehr selten [O'Driscoll und Flanagan, 2016]. In einer Untersuchung innerhalb der verhaltenstherapeutischen Ambulanz der Technischen Universität Dresden wurde untersucht, ob sexuelle Dysfunktionen als sekundäre Diagnose zu einer anderen Achse-I-Störung (z.B. Depression, Angststörung) durch die Behandlung der Primärdiagnose remittieren, selbst wenn sie nicht direkt im Rahmen der Psychotherapie thematisiert werden [Hoyer et al., 2009]. Auf theoretischer Basis erscheint dies plausibel, da verschiedene Störungsbilder die gleichen Risikofaktoren und pathologischen Prozesse beinhalten wie sexuelle Dysfunktionen [Barlow, 1986]. Auf der anderen Seite zeigen Studien, dass sexuelle Dysfunktionen auch unabhängig von anderen Achse-I-Diagnosen vorkommen können [Nicolosi et al., 2004].

Die Untersuchung von Hoyer et al. [2009] an 451 Patienten mit verschiedenen Achse-I-Störungen zeigte zunächst, dass 60\% der Patienten sekundär zu ihrer Primärdiagnose von sexuellen Dysfunktionen berichteten. Bei den Patienten, bei denen die verhaltenstherapeutische Behandlung der Primärdiagnose nicht anschlug, lagen sexuelle Dysfunktionen am häufigsten auch nach Behandlungsende weiterhin vor (48,8\%). Jedoch auch bei den Patienten, bei denen die Therapie zur einer Remission führte oder zumindest teilweise erfolgreich war, bestanden sexuelle Dysfunktionen in $27,1 \%$ bzw. 31,2\% weiter [Hoyer et al., 2009]. Dies deutet darauf hin, dass sexuelle Dysfunktionen zwar in einigen, jedoch nicht in allen Fällen durch die Behandlung einer anderen Achse-IStörung remittieren. Ausstehend ist dabei auch die Frage, wie die Remissionszahlen im Kontext einer PTBS bei einer spezifischen traumafokussierten Psychotherapie sind.

In einer Meta-Analyse von O'Driscoll und Flanagan [2016] wurden sexuelle Funktionsstörungen und deren Ansprechen auf eine traumaspezifische Behandlung analysiert. Dabei zeigte sich, dass eine Erfassung sexueller Beschwerden im Rahmen von Studien zur Psychotherapie der PTBS nur äußerst selten vorgenommen wird, wodurch nur 5 Studien analysiert werden konnten. Die Analyse der Daten ergab, dass sich die Dysfunktionen im Verlauf einer traumaspezifischen Behandlung ohne gezielte Behandlung sexueller Dysfunktionen nicht verbesserten. Zudem wurden sexuelle Dysfunktionen in keiner der Studien nach einem gängigen Diagnosesystem diagnostiziert.

O’Driscoll und Flanagan [2016] schlagen 4 zentrale Elemente zur Intervention bei sexuellen Dysfunktionen im Rahmen einer PTBS-Behandlung vor: 1) Psychoedukation: Hiermit sollen Patien- ten die biologischen Zusammenhänge zwischen PTBS und sexuellem Funktionieren verstehen lernen, Emotionen normalisiert und die Behandlungsmotivation durch einen offenen Umgang mit der Thematik erhöht werden. 2) Entspannungstraining: Dadurch soll die Erwartungsangst verringert und durch eine Beruhigung der Amygdalaaktivität die Wahrscheinlichkeit sexueller Dysfunktionen reduziert werden. 3) Sensate Focus [gemäß Masters und Johnson, 1970]: Sensate Focus beschreibt ein 5-stufiges Vorgehen zur Behandlung sexueller Dysfunktionen, das als Paar durchgeführt wird. Durch Sensate Focus soll langsam die Intimität zum Partner aufgebaut und Angst reduziert werden. Durch ein langsames Vorgehen, das sich zunächst auf Berührungen konzentriert und den Geschlechtsverkehr an sich ausspart, wird das Vertrauen erhöht. Dabei besteht die so genannte «Vetoregel», durch die jegliche Handlungen unterbrochen werden können. Dadurch soll Vermeidungsverhalten abgebaut und die Betroffenen langsam gegenüber angstbesetzten Stimuli exponiert werden, wodurch sich eine Habituation ergeben soll. Dabei können auch Strategien zur kognitiven Umstrukturierung bezüglich negativer Grundannahmen zu Sexualität angewendet werden [O'Driscoll und Flanagan, 2016]. 4) Exposition: Da eine Exposition gegenüber angstbesetzten Situationen in der PTBS-Behandlung als wirksam befunden wurde [Powers et al., 2010] und generell die Methode der Wahl bei Angststörungen ist [Craske, 2015], gilt dies möglichweise auch für den Abbau von Angst vor Sexualität, wobei dazu noch keine Befunde vorliegen.

Denkbar ist auch die Anwendung anderer Strategien aus dem Spektrum der Interventionen bei PTBS, wie kognitive Verfahren aus der Cognitive Processing Therapy [Resick und Schnicke, 1993]. Diese erscheinen hilfreich, da dysfunktionale Annahmen bezüglich Sexualität und des eigenen sexuellen Selbst gezielt angegangen werden können [Müller-Engelmann et al., 2016]. Zusätzlich wären auch Interventionen zur Vermittlung von Selbsthilfe vorstellbar, z.B. durch webbasierte Psychotherapie [Caspar et al., 2013]. Therapiestudien zu Behandlungsformen, die gezielt die Behandlung sexueller Dysfunktionen in die Behandlung der PTBS integrieren, stehen noch aus und wären sinnvoll, um hierzu genauere Aussagen machen zu können. Zudem wäre es wichtig, die Wirksamkeit einzelner Interventionen im Rahmen von Psychotherapiestudien genauer zu untersuchen.

Insgesamt lässt sich sagen, dass sexuelle Dysfunktionen durch die Therapeuten unbedingt angesprochen werden sollten, und zwar nicht nur bei Patienten/innen mit PTBS nach sexueller, sondern auch mit PTBS nach anderen Arten von Traumatisierung. Dann ist im Einzelfall zu entscheiden, ob eine gezielte Behandlung der sexuellen Beschwerden, klinisch und auch subklinisch, indiziert ist. Denkbar wäre, die genannten Schritte an das zweite Drittel der Therapie, also nach der Behandlung der Traumatisierung an sich durch Exposition oder andere Verfahren, anzuschließen. Einige Therapieverfahren schließen dem Expositionsteil Interventionen an, die den Schwerpunkt haben, das «Leben zurückzugewinnen» (z.B. DBT-PTSD [Bohus et al., 2013]) - hier würde sich die Behandlung sexueller Dysfunktionen als Therapiebaustein anbieten. Wichtig ist vor allen Dingen, die Problematik der Patienten/ innen ernst zu nehmen und sie zu ermutigen, offen darüber zu be- 
richten. Hier stellt auch die Psychoedukation und in diesem Kontext insbesondere das Benennen der Häufigkeit der Problematik einen sehr wichtigen und entlastenden Faktor dar. Es ist wichtig, den Patienten/innen zu vermitteln, dass sie nicht die einzigen sind, die an sexuellen Beschwerden leiden, sondern dass es ein häufiges Problem ist, das aber behandelt werden kann. In einzelnen Fällen bieten sich auch Paargespräche an, z.B. um Übungen aus dem Sensate Focus gemeinsam zu besprechen und zu planen. Es ist nicht zu erwarten, dass Patienten/innen ihre sexuellen Beschwerden selbst ansprechen, deshalb ist eine Berücksichtigung der Beschwerden, z.B. auch bereits zu Therapiebeginn im Rahmen der Diagnostik oder im weiteren Verlauf, sehr wichtig. Auf Seiten des Therapeuten ist eine möglichst unverkrampfte, lockere Haltung notwendig, um zu signalisieren: «Es ist ein Thema, über das Sie mit mir reden können, ohne dass ich vor Scham im Boden versinke. Ich nehme Sie und die Problematik ernst.» Dabei ist zu bedenken, dass vielen Patienten/innen das Thema Sexualität sehr unangenehm sein wird und sie umso mehr Entlastung erfahren, je offener der Therapeut damit umgeht. Im vorliegenden Übersichtsartikel wurde explizit nur auf sexuelle Dysfunktionen bei weiblichen PTBS-Patienten eingegangen. Jedoch zeigen verschiedene Befunde, dass sexuelle Dysfunktionen, wie z.B. Erektionsstörungen bei Männern, nach verschiedenen Arten von Traumatisierung ein häufiges Problem sind [Tran et al., 2015; Büttner et al., 2014; Yehuda et al., 2015]. Dies wurde bislang vornehmlich im Bereich von nichtsexueller Traumatisierung festgestellt, wobei sich Studien vor allem zu sexuellen Dysfunktionen bei Kriegsveteranen finden lassen [Tran et al., 2015; Arbanas, 2010]. Insgesamt zeichnen diese Studien ein ähnliches Bild wie bei weiblichen Dysfunktionen im Kontext einer PTBS-Erkrankung, wobei Männer tendenziell häufiger «hypersexuelle Störungen» (Risikosexualität, zwanghafte Sexualität) zeigten als Frauen [Büttner et al., 2014]. Wie auch bei weiblichen PTBSPatienten gibt es bislang keine näheren Empfehlungen oder Manuale dazu, wie sexuelle Beschwerden im Kontext der PTBS behandelt werden sollen. Es liegen sehr wenige Untersuchungen vor, die sexuelle Dysfunktionen bei Männern und Frauen in Abhängigkeit von der Erkrankung an einer PTBS sowie der Art der Traumaerfahrung näher untersucht haben. Insgesamt bleibt der Bereich sexueller Dysfunktionen bei PTBS also ein Themenkomplex, zu dem noch viele offene Fragen, jedoch auch viele potenziell spannende Forschungsfragen bestehen.

\section{Disclosure Statement}

Die Autoren erklären, dass keinerlei Interessenkonflikte bestehen.

\section{Literatur}

American Psychiatric Association: Diagnostic and Statistical Manual of Mental Disorders, ed 5. Arlington, VA, American Psychiatric Publishing, 2013.

Arbanas G: Does post-traumatic stress disorder carry a higher risk of sexual dysfunctions? J Sex Med 2010;7: 1816-1821.

Baldwin DS, Manson C, Nowak M: Impact of Antidepressant Drugs on Sexual Function and Satisfaction. CNS Drugs 2015;29:905-913.

Barlow DH: Causes of sexual dysfunction: the role of anxiety and cognitive interference. J Consult Clin Psychol 1986;54:140-148.

Bartoi MG, Kinder BN: Effects of child and adult sexual abuse on adult sexuality. J Sex Marital Ther 1998;24: 75-90.

Beier KM, Hartmann U, Bosinski HAG: Bedarfsanalyse zur sexualmedizinischen Versorgung. Sexuologie 2000;7: 63-95.

Berner MM, Berner L, Kriston L, et al: Überprüfung der Gültigkeit und Zuverlässigkeit des deutschen Female Sexual Function Index (FSFI-d). Geburtshilfe Frauenheilkd 2004;64:293-303.

Bohus M, Dyer AS, Krüger A, et al: Dialectical behaviour therapy for post-traumatic stress disorder after childhood sexual abuse in patients with and without borderline personality disorder: a randomised controlled trial. Psychother Psychosom 2013;82:221-233.

Bornefeld-Ettmann P, Hoyer J: Strukturiertes Interview für sexuelle Funktionsstörungen nach DSM 5. Teil B Interview. Angepasste Forschungsversion für die Studie «Die Behandlung psychosozialer und neuronaler Folgen von interpersoneller Gewalt in der Kindheit bei Erwachsenen (RELEASE)». Unveröffentlichtes Manuskript. Institut für Psychologie, Technische Universität Dresden, 2014.
Büttner M, Dulz B, Sachsse U, et al: Trauma und sexuelle Störungen: Multizentrische Untersuchung von Patienten mit komplexer posttraumatischer Belastungsstörung. Psychotherapeut 2014;59:385-391.

Caspar F, Berger T, Lotz-Rambaldi W, Hohagen F: Internetbasierte Psychotherapie und E-Mental-Health. Verhaltenstherapie 2013;23:137-139.

Chapman JD: A longitudinal study of sexuality and gynecologic health in abused women. J Am Osteopath Assoc 1989;89:619-624.

Craske MG: Optimizing exposure therapy for anxiety disorders: an inhibitory learning and inhibitory regulation approach. Verhaltenstherapie 2015;25:134-143.

Ehret AM, Berking M: DSM-IV und DSM-5: Was hat sich tatsächlich verändert? Verhaltenstherapie 2013;23: 258-266.

Elklit A, Christiansen DM: ASD and PTSD in rape victims. J Interpers Violence 2010;25:1470-1488.

Foa EB, Kozak MJ: Emotional processing of fear: exposure to corrective information. Psychol Bull 1986:99:20-35.

Friedrich C, Ahrendt HJ: Funktionelle Sexualstörungen der Frau; in Ahrendt H-J, Friedrich C (eds): Sexualmedizin in der Gynäkologie. Berlin, Heidelberg, Springer, 2015, pp 41-63.

Greenberg LS, Korman LM, Paivio SC: Emotion in humanistic psychotherapy; in Cain DJ, Seeman J (eds): Humanistic Psychotherapies: Handbook of Research and Practice. Washington, DC, American Psychological Association, 2002, pp 499-530.

Haase A, Boos A, Schönfeld S, Hoyer J: Sexuelle Dysfunktionen und sexuelle Zufriedenheit bei Patientinnen mit posttraumatischer Belastungsstörung. Verhaltenstherapie 2009;19:161-167.

Hansen NB, Brown LJ, Tsatkin E, et al: Dissociative experiences during sexual behavior among a sample of adults living with HIV infection and a history of childhood sexual abuse. J Trauma Dissociation 2012;13:345-360.
Hayes RD, Dennerstein L, Bennett CM, et al: Risk factors for female sexual dysfunctions in the general population: exploring factors associated with low sexual function and sexual distress. J Sex Med 2008a;5:1681-1693.

Hayes RD, Dennerstein L, Bennett CM, Fairley CK: What is the 'true' prevalence of female sexual dysfunctions and does the way we assess these conditions have an impact? J Sex Med 2008b;5:777-787.

Hayes SC, Wilson KG, Gifford EV, et al: Experiential avoidance and behavioral disorders: a functional dimensional approach to diagnosis and treatment. J Consult Clin Psychol 1996;64:1152-1168.

Heim CM, Mayberg HS, Mletzko T, et al: Decreased cortical representation of genital somatosensory field after childhood sexual abuse. Am J Psychiatry 2013;170: 616-623.

Hofmann S: The future of Cognitive Behavioral Therapy. Verhaltenstherapie 2015;25:40-43.

Houck CD, Nugent NR, Lescano CM, et al: Sexual abuse and sexual risk behavior: beyond the impact of psychiatric problems. J Pediatr Psychol 2010;35:473-483.

Hoyer J: Sexuelle Funktionsstörungen und ambulante Psychotherapie. Psychotherapeut 2013;58:371-378.

Hoyer J, Jahnke S: KfS: Kurzfragebogen zu sexuellen Funktionsstörungen; in Strauß B, Brähler E (eds): Deutschsprachige Verfahren in der Sexualwissenschaft. Göttingen, Hogrefe, 2014, pp 113-116.

Hoyer J, Reitz D, Frank-Noyon E: SISEX: Strukturiertes Interview zu sexuellen Funktionsstörungen; in Strauß B, Brähler E (eds): Deutschsprachige Verfahren in der Sexualwissenschaft. Göttingen, Hogrefe, 2014, pp 173177

Hoyer J, Uhmann S, Rambow J, Jacobi F: Reduction of sexual dysfunction: by-product of cognitive-behavioural therapy for psychological disorders? Sex Relation Ther 2009;24:64-73. 
Jung K, Dyer A, Priebe K, et al: Das Gefühl des Beschmutztseins bei erwachsenen Opfern sexualisierter Gewalt in Kindheit und Jugend. Verhaltenstherapie 2011;21:247-253.

Kaplan HS: The New Sex Therapy. New York, Brunner, Mazel, 1974.

Kennedy SH, Rizvi S: Sexual dysfunction, depression, and the impact of antidepressants. J Clin Psychopharmacol 2009;29:157-164.

Kessler RC, Chiu WT, Demler O: Prevalence, severity, and comorbidity of 12-month DSM-IV disorders in the National Comorbidity Survey Replication. Arch Gen Psychiatry 2005;62:617-627.

Kockott G, Fahrner E-M: Sexualstörungen des Mannes; in Schulte D, Grawe K, Hahlweg K, Vaitl D (eds): Fortschritte der Psychotherapie - Manuale für die Praxis. Göttingen, Hogrefe, 2000, p 2.

Lacelle C, Hébert M, Lavoie F, et al: Sexual health in women reporting a history of child sexual abuse. Child Abuse Negl 2012;36:247-259.

Leonard LM, Follette VM: Sexual functioning in women reporting a history of child sexual abuse: review of the empirical literature and clinical implications. Annu Rev Sex Res 2002;13:346-389.

Letourneau EL, Resnick HS, Kilpatrick DG, et al: Comorbidity of sexual problems and posttraumatic stress disorders in female crime victims. Behav Ther 1996;27: 321-336.

Lewis RW, Fugl-Meyer KS, Corona G, et al: Definitions/ epidemiology/risk factors for sexual dysfunction. J Sex Med 2010;7:1598-1607.

Maercker A, Michael T: Posttraumatische Belastungsstörung; in Margraf J, Schneider S (eds): Lehrbuch der Verhaltenstherapie, ed 3. Heidelberg, Springer, 2009, pp 105-124

Maniglio R: The impact of child sexual abuse on health: a systematic review of reviews. Clin Psychol Rev 2009; 29:647-657.

Masters WH, Johnson VE: Human Sexual Inadequacy. Boston, Little Brown, 1970.

Messman TL, Long PJ: Child sexual abuse and its relationship to revictimization in adult women: a review. Clin Psychol Rev 1996;16:397-420.

Meston CM, Rellini AH, Heiman JR: Women's history of sexual abuse, their sexuality, and sexual self-schemas. J Consult Clin Psychol 2006;74:229-236.
Michael A, O'Keane V: Sexual dysfunction in depression. Hum Psychopharmacol 2000;15:337-345.

Mowrer OH: Learning Theory and Behavior. New York, Wiley, 1960.

Müller-Engelmann M, Dittmann C, Weßlau C, Steil R: Die Cognitive Processing Therapie - Cognitive Therapy Only zur Behandlung der komplexen Posttraumatischen Belastungsstörung. Verhaltenstherapie 2016;26: 195-203.

Najman JM, Dunne MP, Purdie DM, et al: Sexual abuse in childhood and sexual dysfunction in adulthood: an Australian population-based study. Arch Sex Behav 2005;34:517-526.

Nicolosi A, Moreira ED, Villa M, Glasser DB: A population study of the association between sexual function, sexual satisfaction and depressive symptoms in men. J Affect Disord 2004;82:235-243.

O'Driscoll C, Flanagan E: Sexual problems and post-traumatic stress disorder following sexual trauma: a metaanalytic review. Psychol Psychother 2016;89:351-367.

Payne KA, Binik YM, Amsel R, Khalifé S: When sex hurts, anxiety and fear orient attention towards pain. Eur J Pain 2005;9:427-427.

Polusny MA, Follette VM: Long-term correlates of child sexual abuse: theory and review of the empirical literature. Appl Prev Psychol 1995;4:143-166.

Powers MB, Halpern JM, Ferenschak MP, et al: A metaanalytic review of prolonged exposure for posttraumatic stress disorder. Clin Psychol Rev 2010;30:635641.

Raina R, Pahlajani G, Khan S, et al: Female sexual dysfunction: classification, pathophysiology, and management. Fertil Steril 2007;88:1273-1284.

Reinecke A, Schöps D, Hoyer J: Sexuelle Dysfunktionen bei Patienten einer verhaltenstherapeutischen Hochschulambulanz: Häufigkeit, Erkennen, Behandlung. Verhaltenstherapie 2006;16:166-172.

Resick PA, Schnicke M: Cognitive Processing Therapy for Rape Victims: A Treatment Manual, vol 4. Newbury Park, Sage Publications, Inc, 1993.

Sarwer DB, Durlak JA: Childhood sexual abuse as a predictor of adult female sexual dysfunction: a study of couples seeking sex therapy. Child Abuse Negl 1996;20: 963-972.

Schauer M, Elbert T: Dissociation following traumatic stress: etiology and treatment. J Psychol 2010;218: $109-127$.
Scheiderer EM, Wood PK, Trull TJ: The comorbidity of borderline personality disorder and posttraumatic stress disorder: revisiting the prevalence and associations in a general population sample. Borderline Personal Disord Emot Dysregul 2015;2:11.

Senn TE, Carey MP, Vanable PA: Childhood and adolescent sexual abuse and subsequent sexual risk behavior: evidence from controlled studies, methodological critique, and suggestions for research. Clin Psychol Rev 2008;28:711-735.

Shapiro BL, Schwarz JC: Date rape: its relationship to trauma symptoms and sexual self-esteem. J Interpers Violence 1997;12:407-419.

Shifren JL, Monz BU, Russo PA, et al: Sexual problems and distress in United States women: prevalence and correlates. Obstet Gynecol 2008;112:970-978.

Stephenson KR, Meston CM: Why is impaired sexual function distressing to women? The primacy of pleasure in female sexual dysfunction. J Sex Med 2015;12:728-737.

Stephenson KR, Pulverman CS, Meston CM: Assessing the association between childhood sexual abuse and adult sexual experiences in women with sexual difficulties. J Trauma Stress 2014;27:274-282.

Stoffels H, Ernst C: Erinnerung und Pseudoerinnerung: Über die Sehnsucht, Traumaopfer zu sein. Nervenarzt 2002;73:445-451.

Tagay S, Herpertz S, Langkafel M, Senf W: Trauma, posttraumatische Belastungsstörung und Somatisierung. Psychother Psych Med 2004;54:198-205.

Tran JK, Dunckel G, Teng EJ: Sexual dysfunction in veterans with post-traumatic stress disorder. J Sex Med 2015;12:847-855.

Ullman S: Talking about Sexual Assault: Society's Response to Survivors. Washington, DC, American Psychological Association, 2010.

Van Berlo W, Ensink B: Problems with sexuality after sexual assault. Annu Rev Sex Res 2000;11:235-257.

Van Bruggen LK, Runtz MG, Kadlec H: Sexual revictimization: the role of sexual self-esteem and dysfunctional sexual behaviors. Child Maltreat 2006;11:131-145.

World Health Organization: International Statistical Classification of Diseases and Related Health Problems, 10th Revision (ICD-10). Geneva, WHO, 1992.

Yehuda R, Lehrner A, Rosenbaum TY: PTSD and Sexual Dysfunction in Men and Women. J Sex Med 2015;12 1107-1119. 